\title{
Determination of the Thermodynamic Scaling Exponent for Relaxation in Liquids from Static Ambient-Pressure Quantities
}

\author{
R. Casalini and C. M. Roland \\ Chemistry Division, Naval Research Laboratory, Code 6120, Washington, D.C. 20375-5342, USA
}

(Received 5 March 2014; published 18 August 2014)

\begin{abstract}
An equation is derived that expresses the thermodynamic scaling exponent, $\gamma$, which superposes relaxation times $\tau$ and other measures of molecular mobility determined over a range of temperatures and densities, in terms of static physical quantities. The latter are available in the literature or can be measured at ambient pressure. We show for 13 materials, both molecular liquids and polymers, that the calculated $\gamma$ are equivalent to the scaling exponents obtained directly by superpositioning. The assumptions of the analysis are that the glass transition $T_{g}$ is isochronal (i.e., $\tau_{\alpha}$ is constant at $T_{g}$, which is true by definition) and that the pressure derivative of the glass temperature is given by the first Ehrenfest relation. The latter, derived assuming continuity of the entropy at the glass transition, has been corroborated for many glass-forming materials at ambient pressure. However, we find that the Ehrenfest relation breaks down at elevated pressure; this limitation is of no consequence herein, since the appeal of the new equation is its applicability to ambient-pressure data. The ability to determine, from ambient-pressure measurements, the scaling exponent describing the high-pressure dynamics extends the applicability of this approach to a broader range of materials. Since $\gamma$ is linked to the intermolecular potential, the new equation thus provides ready access to information about the forces between molecules.
\end{abstract}

DOI: 10.1103/PhysRevLett.113.085701

PACS numbers: $64.70 . \mathrm{kj}, 78.35 .+\mathrm{c}$

An important development in understanding the dynamics of supercooled liquids was the discovery that relaxation times $\tau$, viscosities $\eta$, diffusion constants $D$, and other measures of molecular mobility can be expressed as a function of the product of temperature $T$ and specific volume $V$ with the latter raised to a material constant $[1,2,3]$. Thus, for the relaxation time,

$$
\tau=f\left(T V^{\gamma}\right),
$$

where $f$ is a function that is unknown a priori; similar equations can be written for the other dynamic properties. Equation (1) has been experimentally validated for more than 100 liquids and polymers, with data from dielectric spectroscopy, dynamic light scattering, and viscosity measurements superposing when plotted versus $T V^{\gamma}[4,5]$. The only materials exhibiting deviations from Eq. (1) are those that undergo changes in chemical structure, such as their degree of hydrogen bonding, with change in state point [6]. Thermodynamic scaling has also been applied to results from molecular dynamics (MD) simulations [7], and Eq. (1) is found to be accurate for realistic densities, although for extremes in $V$ some variation in $\gamma$ is necessary for accurate superpositioning [8]. MD simulations have also identified other characteristics of liquids related to thermodynamic scaling [9]. Prominent among these is the strong correlation of fluctuations in the virial pressure $(W)$ with fluctuations of the potential energy $(U)$; the proportionality constant is equal to the thermodynamic scaling exponent, $d W / d U=\gamma[10,11]$.
One consequence of $d W-d U$ correlation is it provides a route to the calculation of $\gamma$ from linear thermoviscoelastic response functions, specifically (the dynamic components of) the compressibility, heat capacity, expansion coefficient, and shear modulus [12]. The method obviates the need for measurements at elevated pressures or densities; $\gamma$ can be determined from measurements at one ambient-pressure temperature. This approach has been demonstrated for a silicone oil at $214 \mathrm{~K}$ [12]; the results, $\gamma=6 \pm 2$, were consistent with the scaling exponent determined from superposition of $\tau(T, V), \gamma=6.2 \pm 0.2$. The limitation of the method is the difficulty of accurate measurements of frequency-dependent thermoviscoelastic response functions.

A route to $\gamma$ that does not entail any dynamic measurements takes advantage of the fact that the relaxation time at the glass transition is constant (this is true by definition in a kinetic interpretation of vitrification). It follows that the ratio $T_{g} V_{g}^{\gamma}$ is constant, where the subscript refers to the pressure-dependent glass transition. The scaling exponent is determined as [4]

$$
\gamma=-\left(\partial \log T_{g} / \partial \log V_{g}\right)_{P}
$$

Equation (2) has particular significance in the study of liquid crystals because the bracketed quantity (known as the thermodynamic potential parameter with the subscript " $g$ " denoting a phase transition), is central to models of the phase stability of liquid crystals [13]. Conformance of the $\gamma$ from Eq. (2) with the value obtained by 
superpositioning rotational relaxation times of liquid crystals indicates constancy of the latter at the clearing line (i.e., at state points demarcating the nematic-isotropic phase transition) $[14,15]$.

A general use of thermodynamic scaling is to provide a means to efficiently categorize relaxation data obtained over a broad range of thermodynamic conditions. More significantly, $\gamma$ is a measure of the relative roles of thermal energy and density in governing the dynamics; for example, the scaling exponent can be related to the activation energy ratio [16]

$$
E_{V} / H_{P}=\left(1+\alpha_{P} T_{g} \gamma\right)^{-1},
$$

in which $E_{V}=\left.R\left(\partial \ln \tau / \partial T^{-1}\right)\right|_{\rho}$ is the isochoric activation energy and $H_{P}=\left.R\left(\partial \ln \tau / \partial T^{-1}\right)\right|_{P}$ the activation enthalpy at constant pressure. (These are actually "apparent" activation energies, since neither $E_{V}$ nor $H_{P}$ are temperature invariant.) $\alpha_{P}$ is the isobaric thermal expansion coefficient. The advantage of $\gamma$ is that it is a material constant, whereas $E_{V} / H_{P}$ varies with state point [4]. An intriguing finding from MD simulations is the connection between the magnitude of $\gamma$ and the steepness of the intermolecular potential in the region around the mean separation distance of Lennard-Jones particles [17,18,19,20,21]. Of practical utility is that knowledge of $\gamma$ enables $\tau$ (or $\eta, D, \ldots$ ) to be calculated for any thermodynamic condition from measurements at only ambient pressure. However, what has heretofore been lacking is a way to quantify $\gamma$ without the necessity of carrying out experiments at elevated pressure or making relaxation measurements. In this Letter we describe a procedure to accomplish this.

Naoki [22] derived an expression for the thermal pressure coefficient in terms of the activation energy ratio,

$$
\left.\frac{\partial T}{\partial P}\right|_{\tau}=\left.\left(1-\frac{E_{V}}{H_{P}}\right) \frac{\partial T}{\partial P}\right|_{V} .
$$

This equation follows directly from the assumption that relaxation is an activated process described by an Arrhenius-type equation, which is true if only state points corresponding to $T_{g}$ are considered. Equations (3) and (4) can be combined to yield for the scaling exponent,

$$
\gamma=\frac{\left.\left(T_{g} \kappa_{T}\right)^{-1} \frac{\partial T}{\partial P}\right|_{\tau}}{1-\left.\frac{\partial T}{\partial P}\right|_{\tau} \frac{\alpha_{P}}{\kappa_{T}}},
$$

where $\kappa_{T}$ is the isothermal compressibility. (Note that while $\kappa_{T}$ is a pressure derivative, its ambient pressure value can be obtained without measurements at elevated pressure, for example, by calculating it from the adiabatic compressibility measured by Brillouin scattering.) Continuity of the entropy at the glass transition yields [23,24]

$$
\left.\frac{\partial T}{\partial P}\right|_{\tau}=\frac{\Delta \alpha_{P} T V}{\Delta c_{P}}
$$

in which $c_{p}$ is the specific heat, and the $\Delta$ 's denote the change at the glass transition. When substituted in Eq. (5), Eq. (6), known as the first Ehrenfest relation, gives

$$
\gamma=V \Delta \alpha_{P} /\left(\Delta c_{P} \kappa_{T}-T V \alpha_{P} \Delta \alpha_{P}\right) .
$$

Equation (7), our main result, expresses the scaling exponent in terms of physical units that can be measured at ambient pressure, without relaxation measurements. Equation (2) also yields $\gamma$ without the need for dynamic measurements, but it requires the glass transition temperature to be determined at elevated pressure. Equation (7) involves only ambient pressure quantities.

There are two assumptions underlying Eq. (7): at $T_{g} \tau$ is constant and the entropy is continuous. As stated, the first assumption is true by definition, although the fact that the glass transition is a kinetic phenomenon means that the values of some parameters in Eq. (7) are sensitive to thermal and pressure histories; this potentially introduces uncertainty into the calculated $\gamma$. The second assumption has been verified, at least at ambient pressure, for many glass formers [25-30].

We test Eq. (7) by comparing the computed $\gamma$ to values obtained in the usual fashion by superposition of relaxation times. This is done for 13 liquids for which the thermodynamic data in Eq. (7) are available (Table I) [12,31-44]. The results, shown in Fig. 1, affirm the correctness of the new expression for $\gamma$.

Although the present analysis allows $\gamma$ to be determined from ambient-pressure data, the quantities in Eq. (7) can also be measured at elevated pressures. For normal liquids ("correlating liquids" in the parlance of Ref. [45]) $\gamma$ is constant, so the results would be the same. However, for liquids with structure that changes with temperature or pressure, such as the concentration of $\mathrm{H}$ bonds, $\gamma$ is expected to vary. Equation (7) cannot be applied in such situations because of the limited validity of Eq. (6) at high pressures. This is illustrated in Fig. 2 for polyvinylacetate using the equations of state for the liquid and glass from Ref. [39]. The pressure dependence of $\Delta c_{p}$ was calculated assuming the heat capacity of the glass is constant and that of the liquid varies as $\int_{0}^{P} T\left(\partial V^{2} / \partial T^{2}\right) d x$. Although Eq. (6) is accurate at atmospheric pressure, it underestimates $\partial T /\left.\partial P\right|_{\tau}$ at higher pressures. [This deviation of Eq. (6) at elevated $P$ appears to be a new observation.] We also found that for sorbitol (data not shown) the departure of $\partial T /\left.\partial P\right|_{\tau}$ from experimental data was as much as $50 \%$ at $100 \mathrm{MPa}$.

We previously showed [40] that the thermodynamic scaling property can be derived from models that connect the supercooled dynamics to the entropy [46]. These also provide an alternative route to Eq. (7). To show this we note that the configurational entropy $S_{C}$ conforms to thermodynamic scaling [47,48] (Fig. 3), 
TABLE I. Physical quantities used to calculate the scaling exponent.

\begin{tabular}{lccccccccccc}
\hline \hline & $\begin{array}{c}\alpha_{P}^{\text {liq }} \\
\left(10^{4} \mathrm{~K}^{-1}\right)\end{array}$ & $\begin{array}{c}\Delta \alpha_{P} \\
\left(10^{4} \mathrm{~K}^{-1}\right)\end{array}$ & $\begin{array}{c}\Delta c_{P}{ }^{\mathrm{a}} \\
\left(\mathrm{Jmol}^{-1} \mathrm{~K}^{-1}\right)^{\mathrm{a}}\end{array}$ & $\begin{array}{c}V \\
\left(\mathrm{~cm}^{3} \mathrm{~mol}^{-1}\right)\end{array}$ & $\begin{array}{c}\kappa_{T} \\
\left(10^{4} \mathrm{MPa}^{-1}\right)\end{array}$ & $\begin{array}{c}T_{g} \\
(\mathrm{~K})\end{array}$ & $\begin{array}{c}\left.\frac{\partial T}{\partial P}\right|_{\tau} ^{\mathrm{b}} \\
\left(\mathrm{KMPa}^{-1}\right)\end{array}$ & $\gamma$ & $\begin{array}{l}\gamma \\
\gamma_{\exp }\end{array}$ & $\mathrm{Reference}$ \\
\hline sorbitol & 4.46 & 2.74 & 189.4 & 111.7 & 11.5 & 272 & 0.044 & $0.14 \pm 0.012$ & 0.16 & {$[30]$} \\
glycerol & 4.8 & 2.4 & 81 & 69.99 & 1.8 & 183 & 0.0379 & $1.28 \pm 0.15$ & $1-1.6$ & {$[31]$} \\
A & 5.24 & 2.9 & 37.2 & $86.96^{\mathrm{c}}$ & 3.9 & 380 & 0.258 & $2.8 \pm 0.34$ & 1.8 & {$[32]$} \\
o-PMMA & 6.2 & 3.8 & 43.1 & 84.22 & 4.85 & 338.9 & 0.251 & $2.3 \pm 0.2$ & 1.94 & {$[33,34]$} \\
PS & 6.0 & 3.2 & 34 & 100.8 & 6.5 & 353 & 0.328 & $2.1 \pm 0.3$ & 2.5 & {$[35,36]$} \\
PCHMA & 5.36 & 1.5 & 33.6 & 161.5 & 4.7 & 336 & 0.245 & $2.1 \pm 0.4$ & 2.5 & {$[37]$} \\
PVAc & 7.15 & 4.52 & 40.7 & 72.5 & 5.0 & 304 & 0.245 & $2.48 \pm 0.14$ & 2.5 & {$[38,39]$} \\
PMA & 6.64 & $3.34^{\mathrm{c}}$ & 37.8 & 70.28 & 3.8 & 287 & 0.201 & $2.83 \pm 0.3$ & 2.55 & {$[40]$} \\
OTP & 7.08 & 5.49 & 113 & 205.9 & 4.2 & 246 & 0.246 & $4.05 \pm 0.3$ & 4 & {$[41]$} \\
PDE & 6.08 & 3.16 & 96.8 & 255.07 & 3.64 & 298 & 0.248 & $3.91 \pm 0.4$ & 4.5 & {$[39,42]$} \\
OTP-OPP & 8.5 & 5.6 & 123 & 203.9 & 3.4 & 233.7 & 0.217 & $6.0 \pm 0.3$ & 6.2 & {$[35]$} \\
DC704 & 4.6 & 3.5 & 145.4 & 425.18 & 2.5 & 212 & 0.217 & $6.8 \pm 0.8$ & 6.2 & {$[12]$} \\
PCB & 7.50 & 3.4 & 66.1 & 239.6 & 4.0 & 268.9 & 0.330 & $8.1 \pm 0.86$ & 8.5 \\
\hline \hline
\end{tabular}

aper repeat unit for polymers.

bcalculated using Eq. (6).

${ }^{\mathrm{c}} \alpha_{P}^{\text {glass }}$ taken at $200 \mathrm{MPa}$.

$$
S_{c}=g\left(T V^{\gamma}\right)
$$

where $g$ is a function, and the scaling exponent is the same $\gamma$ as in Eq. (1). From the continuity of the entropy at the glass transition, the derivatives of temperature and volume are related as

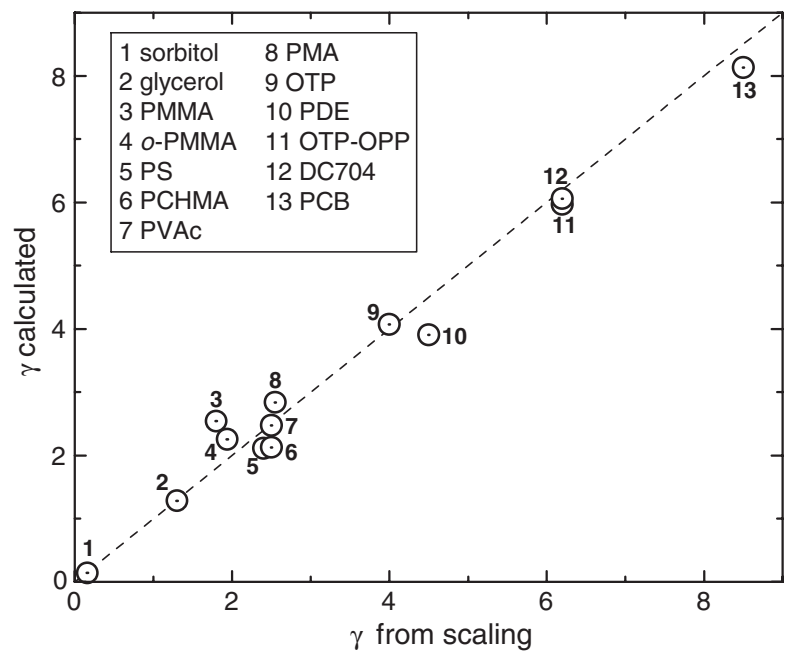

FIG. 1. Scaling exponent calculated from Eq. (7) versus the value of $\gamma$ obtained by superposition of relaxation times. PMMA, polymethymethacrylate (high polymer); o-PMMA, oligomeric PMMA; PS, polystyrene; PCHMA, polycyclohexylmethacrylate; PVAC, polyvinylacetate; PMA, polymethacrylate; OTP, orthoterphenyl; PED, phenolphthalein dimethylether; DC704, tetramethyl tetraphenyl trisiloxane; OTP-OPP, mixture of $67 \%$ OTP and 33\% ortho-phenylphenol; PCB, chlorinated biphenyl. The dashed line represents equivalence of the $\gamma$. References for the data are in Table I.

$$
\begin{aligned}
& \left(\left.\frac{\partial S^{\text {liquid }}}{\partial T}\right|_{V}-\left.\frac{\partial S^{\text {glass }}}{\partial T}\right|_{V}\right) d T \\
& =-\left(\left.\frac{\partial S^{\text {liquid }}}{\partial V}\right|_{T}-\left.\frac{\partial S^{\text {glass }}}{\partial V}\right|_{T}\right) d V
\end{aligned}
$$

Because the nonconfigurational component of the entropy is unaffected by vitrification [40,46], it cancels out and Eq. (9) can be rewritten as

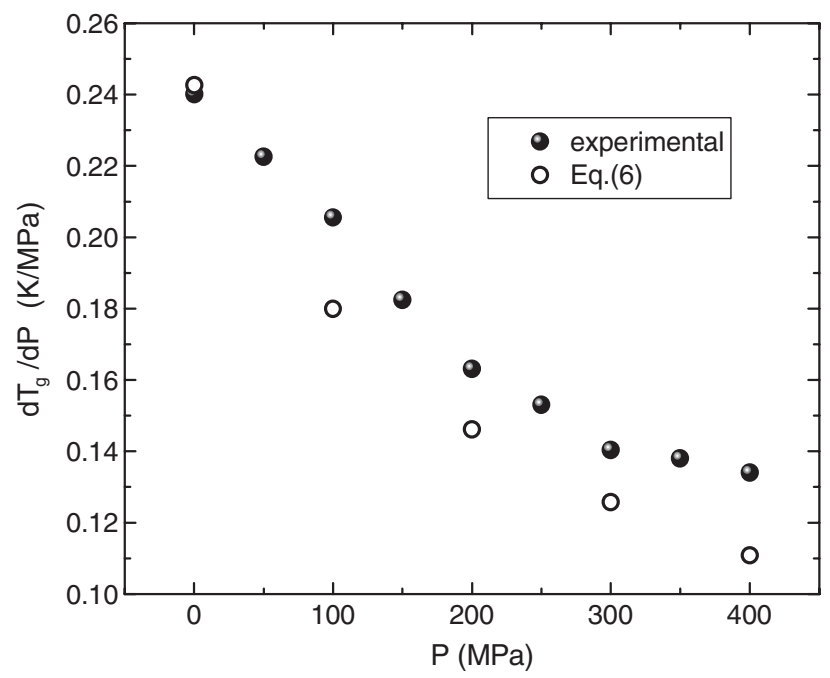

FIG. 2. Thermal pressure coefficient at $T_{g}$ as a function of pressure determined experimentally (filled symbols) and calculated using the first Ehrenfest relation (open symbols). The latter's validity is limited to ambient pressure. 


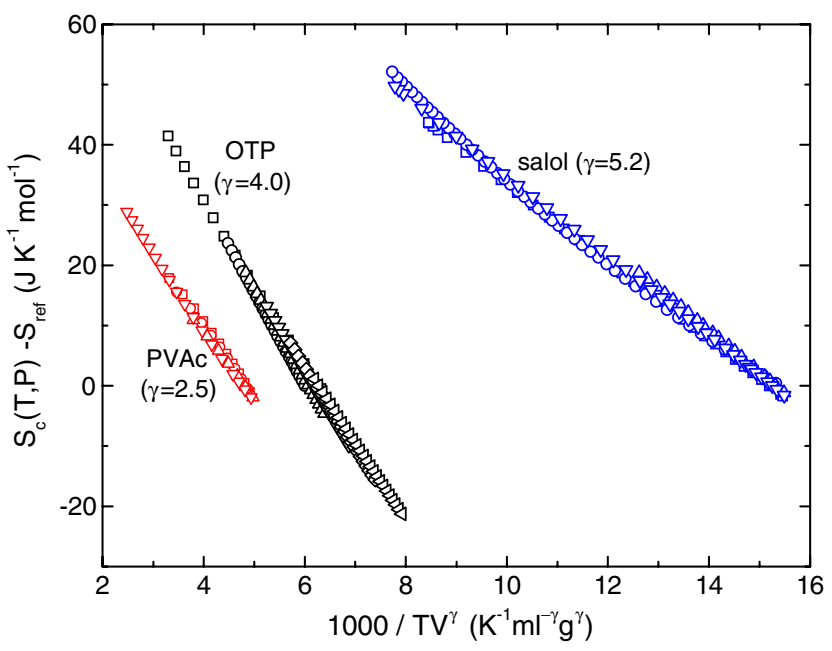

FIG. 3 (color online). Configurational entropy of three liquids measured at various temperatures and pressures plotted versus the scaling variable with the indicated value of $\gamma$. The plotted values are at a reference point $S_{\text {ref }}$ after subtraction of $S_{c}$; thus, the ordinate can be negative (see Refs. [46,47]).

$$
\begin{aligned}
& \left(\left.\frac{\partial S_{C}^{\text {liquid }}}{\partial T}\right|_{V}-\left.\frac{\partial S_{C}^{\text {glass }}}{\partial T}\right|_{V}\right) d T \\
& =-\left(\left.\frac{\partial S_{C}^{\text {liquid }}}{\partial V}\right|_{T}-\left.\frac{\partial S_{C}^{\text {glass }}}{\partial V}\right|_{T}\right) d V .
\end{aligned}
$$

It follows from the scaling property of $S_{C}$ [Eq. (8)] that

$$
\left.\frac{\partial S_{\text {conf }}}{\partial V}\right|_{T}=\left.\gamma \frac{T}{V} \frac{\partial S_{\text {conf }}}{\partial T}\right|_{V}
$$

Equation (11) bears a formal similarity to the relation between the Grüneisen parameter and the derivatives of $S$ [40]. Combining Eqs. (10) and (11) yields

$$
\left.\frac{\partial V}{\partial T}\right|_{\tau}=-\frac{V}{T \gamma} .
$$

Rewriting the temperature derivative of the volume at constant $\tau$ as

$$
\left.\frac{\partial V}{\partial T}\right|_{\tau}=\left.\frac{\partial V}{\partial T}\right|_{P}+\left.\left.\frac{\partial V}{\partial P}\right|_{T} \frac{\partial P}{\partial T}\right|_{\tau}
$$

it can be seen that Eq. (12) is equivalent to Eq. (5), and thus Eq. (7) is obtained.

Since the discovery of thermodynamic scaling $[1,2,3]$ much experimental and theoretical work has been done to relate the exponent $\gamma$ to physical properties of the materials [4,5], such as activation energies for the dynamics [16] and the steepness of the intermolecular potential $[17,18,19,20]$. There also exist expressions relating certain thermodynamic properties and their changes at the liquid-glass transition. An example of the latter is the first Ehrenfest equation, which has been corroborated for a large number of materials $[25,26,27,28,29,30]$. Combining this equation with a formula for $\gamma$, we derive an expression for the scaling exponent in terms of static physical properties. This expression also follows from thermodynamic scaling of the configurational entropy. The predictions of this new relation are found to be in agreement with results obtained directly by superposition of relaxation data. This analysis enables dynamic properties to be determined for any thermodynamic condition from quantities that are routinely measured or available in the open literature. The fact that the accuracy of the first Ehrenfest equation appears to be limited to low pressures means that the analysis can only be implemented using ambient-pressure quantities; however, this limitation is inconsequential, since the advantage of the method is obviating the requirement for high-pressure measurements. Of course Eq. (7) can only be applied to glass-forming materials. However, the insights provided into the liquid structure, the intermolecular potential, and their relations to dynamics are of broader significance. Thus, this new development serves as an example of how research into the glass transition can contribute more generally to condensed matter science.

This work was supported by the Office of Naval Research.

[1] R. Casalini and C. M. Roland, Phys. Rev. E 69, 062501 (2004).

[2] C. Dreyfus, A. Le Grand, J. Gapinski, W. Steffen, and A. Patkowski, Eur. Phys. J. B 42, 309 (2004).

[3] C. Alba-Simionesco, A. Cailliaux, A. Alegria, and G. Tarjus, Europhys. Lett. 68, 58 (2004).

[4] C. M. Roland, S. Hensel-Bielowka, M. Paluch, and R. Casalini, Rep. Prog. Phys. 68, 1405 (2005).

[5] C. M. Roland, Viscoelastic Behavior of Rubbery Materials (Oxford University Press, New York, 2011).

[6] C. M. Roland, R. Casalini, R. Bergman, and J. Mattsson, Phys. Rev. B 77, 012201 (2008).

[7] L. Bohling, T. S. Ingebrigtsen, A. Grzybowski, M. Paluch, J. C. Dyre, and T. B. Schroder, New J. Phys. 14, 113035 (2012), and references therein.

[8] N. P. Bailey, L. Bohling, A. A. Veldhorst, T. B. Schroder, and J. C. Dyre, J. Chem. Phys. 139, 184506 (2013).

[9] J. P. Dyre, J. Phys. Chem. B, doi: 10.1021/jp501852b (2014).

[10] N. P. Bailey, U. R. Pedersen, N. Gnan, T. B. Schroder, and J. C. Dyre, J. Chem. Phys. 129, 184507 (2008); 129, 184508 (2008).

[11] D. Coslovich and C. M. Roland, J. Chem. Phys. 130, 014508 (2009).

[12] D. Gundermann, U. R. Pedersen, T. Hecksher, N. P. Bailey, B. Jakobsen, T. Christensen, N. B. Olsen, T. B. Schroder, D. Fragiadakis, R. Casalini, C. M. Roland, J. C. Dyre, and K. Niss, Nat. Phys. 7, 816 (2011). 
[13] J. R. McColl and C. S. Shih, Phys. Rev. Lett. 29, 85 (1972); J. R. McColl, Phys. Lett. 38A, 55 (1972).

[14] S. Urban and C. M. Roland, J. Non-Cryst. Solids 357, 740 (2011).

[15] C. M. Roland, Soft Matter 4, 2316 (2008).

[16] R. Casalini and C. M. Roland, Phys. Rev. B 71, 014210 (2005).

[17] C. M. Roland, S. Bair, and R. Casalini, J. Chem. Phys. 125, 124508 (2006).

[18] D. Coslovich and C. M. Roland, J. Phys. Chem. B 112, 1329 (2008).

[19] U. R. Pedersen, N. P. Bailey, T. B. Schrøder, and J. C. Dyre, Phys. Rev. Lett. 100, 015701 (2008).

[20] U. R. Pedersen, T. B. Schroder, and J. C. Dyre, Phys. Rev. Lett.. 105, 157801 (2010).

[21] A. Grzybowski, K. Koperwas, and M. Paluch, Phys. Rev. E 86, 031501 (2012).

[22] M. Naoki, H. Endou, and K. Matsumoto, J. Phys. Chem. 91, 4169 (1987).

[23] J. Jäckle, Rep. Prog. Phys. 49, 171 (1986).

[24] G. B. McKenna, J. Non-Cryst. Solids 355, 663 (2009).

[25] J. M. O’Reilly, J. Polym. Sci. 57, 429 (1962).

[26] M. Goldstein, J. Chem. Phys. 39, 3369 (1963).

[27] G. Gee, Polymer 7, 177 (1966).

[28] T. Atake and C. A. Angell, J. Phys. Chem. 83, 3218 (1979).

[29] Th. M. Nieuwenhuizen, Phys. Rev. Lett. 79, 1317 (1997).

[30] R. Casalini, R. F. Gamache, and C. M. Roland, J. Chem. Phys. 135, 224501 (2011).

[31] M. Naoki, K. Ujita, and S. Kashima, J. Phys. Chem. 97, 12356 (1993).

[32] E. Donth, The Glass Transition (Springer-Verlag, Berlin, 2001), p. 224.
[33] S. Theobald, W. Pechhold, and B. Stoll, Polymer 42, 289 (2001).

[34] R. Casalini and C. M. Roland, Macromolecules 46, 6364 (2013).

[35] R. Casalini, C. M. Roland, and S. Capaccioli, J. Chem. Phys. 126, 184903 (2007).

[36] S. Takahara, M. Ishikawa, O. Yamamuro, and T. Matsuo, J. Phys. Chem. B 103, 792 (1999).

[37] C. M. Roland, Macromolecules 43, 7875 (2010).

[38] C. M. Roland and R. Casalini, Macromolecules 40, 3631 (2007).

[39] J.E. McKinney and R. Simha, Macromolecules 7, 894 (1974).

[40] R. Casalini, U. Mohanty, and C. M. Roland, J. Chem. Phys. 125, 014505 (2006).

[41] R. Casalini, D. Fragiadakis, and C. M. Roland, Macromolecules 44, 6928 (2011).

[42] T. Atake and C. A. Angell, J. Phys. Chem. 83, 3218 (1979).

[43] D. Cangialosi, A. Alegría, and J. Colmenero Phys. Rev. E 80, 041505 (2009).

[44] C. M. Roland and R. Casalini, J. Chem. Phys. 122, 134505 (2005).

[45] N. P. Bailey, T. Christensen, B. Jakobsen, K. Niss, N. B. Olsen, U. R. Pedersen, T. B. Schroder, and J. C. Dyre, J. Phys. Condens. Matter 20, 244113 (2008).

[46] I. Avramov, J. Non-Cryst. Solids 262, 258 (2000); 351, 3163 (2005).

[47] R. Casalini and C. M. Roland, Philos. Mag. 87, 459 (2007).

[48] C. M. Roland and R. Casalini, J. Phys. Condens. Matter 19, 205118 (2007). 